\title{
Circle-criterion Based Nonlinear Observer Design for Sensorless Induction Motor Control
}

\author{
Wafa Bourbia $^{1} \quad$ Farid Berrezzek ${ }^{2} \quad$ Bachir Bensaker $^{3}$ \\ ${ }^{1}$ University Badji Mokhtar, Department of Electrotechnic, BP. 12, Annaba 23000, Algeria \\ ${ }^{2}$ University Med Cherif Messadia, Souk Ahras 41000, Algeria \\ ${ }^{3}$ Laboratory of Electromechanical Systems, University Badji Mokhtar, BP.12, Annaba 23000 Algeria
}

\begin{abstract}
This paper deals with the design of a nonlinear observer for sensorless induction motor control. Based upon the circle criterion approach, a nonlinear observer is designed to estimate pertinent but unmeasurable state variables of the considered induction machine for sensorless control purpose. The observer gain matrices are computed as a solution of linear matrix inequalities (LMI) that ensure the stability conditions of the state observer error dynamics in the sense of Lyapunov concepts. Measured and estimated state variables can be exploited to perform a state feedback control of the machine system. The simulation results are presented to illustrate the effectiveness of the proposed approach for nonlinear observer design.
\end{abstract}

Keywords: Nonlinear observer, circle-criterion, Lyapunov stability, linear matrix inequalties (LMI), induction motor.

\section{Introduction}

It is well known that induction motor is one of the most widely used machines in industrial applications. This is due to its high reliability, relatively low cost, and modest maintenance requirements. However, induction motor is also known as a complex nonlinear system, in which timevarying parameters entail additional difficulties for machine control, conditions monitoring and fault diagnostic purposes $^{[1]}$. Furthermore, only a few state variables of the machine are available for on line measurement because of technical and/or economical constraints of the considered application.

In order to perform advanced control techniques, there is a great need of a reliable and accurate estimation of the unmeasurable key state variables of the machine. A state estimator, also called state observer, is a dynamic system that is driven by the inputs and outputs of the considered system, and estimates asymptotically its unmeasurable state variables. It is a "soft sensor" that plays an important role not only in sensorless control techniques but also in conditions monitoring, fault diagnosis, predictive maintenance and fault tolerant control techniques ${ }^{[1-3]}$.

A control literature review shows that nonlinear state observer design approaches can be roughly divided into three classes ${ }^{[4-9]}$. The first class of approaches attempts to eliminate the system nonlinearities by a technique of linearization $^{[3]}$. Its drawback is a set of extremely restrictive conditions that can hardly be met by a physical system. The second class of approaches attempts to dominate the system nonlinearities by using a high gain output correction term $^{[4,5]}$. Its drawbacks are the block triangular structure, the destabilizing effect of the peaking phenomenon, and the

Regular paper

Special Issue on Recent Advances on Complex Systems Control, Modelling and Prediction II

Manuscript received February 13, 2013; accepted December 2, 2013 sensitivity against measurement noises.

The third class of approaches to design nonlinear observers exploits the system nonlinearity. Lipschitz and sector properties are the main nonlinearity properties that are exploited $^{[6,10-12]}$

In order to benefit from some recent advances in nonlinear observer based control developed by the control community, in this paper we focus our attention on the application of one method of the third class, the so-called circlecriterion approach, to design a nonlinear observer for induction motor sensorless control. The circle criterion approach is a new line of research introduced for continuous-time systems by [10]. It permits to handle directly the system nonlinearities with less restriction than linearization and high gain approaches ${ }^{[10,11]}$. A detailed proof of the main theorem is given in this paper.

The paper is organized as follows: In the second section, we present the ingredient of nonlinearity satisfying the sector properties and the design of the nonlinear observer. The considered nonlinear induction motor model is presented in the third section. In the fourth section, we present simulation results and comments. A conclusion ends the paper.

\section{Circle criterion based nonlinear ob- server design}

In contrast to the linearization based and high-gain approaches which attempt to eliminate the system nonlinearities using a nonlinear state transformation or to dominate them by a high gain term of correction, circle-criterion approach exploits the type of system nonlinearities to design nonlinear observer. In its basic form introduced by Arcak and Kokotovic $^{[10]}$, the approach is applicable to a class of nonlinear systems that can be decomposed in linear and nonlinear parts with a condition that the nonlinearities satisfy the sector property. 


\subsection{Basic sector properties}

A nonlinear function $f(z, t)$ such as $f(z, t):[0+\infty] \times$ $\mathbf{R}^{p} \rightarrow \mathbf{R}^{p}$ is said to belong to the sector $[0+\infty]$ if $z f(z, t) \geqslant 0$.

This relation is known as the sector property of a nonlinear function. It is equivalent to the following relation:

$$
\left(v_{1}-v_{2}\right)\left[f\left(v_{1}, t\right)-f\left(v_{2}, t\right)\right] \geqslant 0, \quad \forall v_{1}, v_{2} \in \mathbf{R}^{+}
$$

here $v_{1}-v_{2}=z$ and $\left[f\left(v_{1}, t\right)-f\left(v_{2}, t\right)\right]=f(z, t)$, where $v_{1}$ and $v_{2}$ are two real positive numbers.

Relation (1) states that the nonlinear function $f(z, t)$ is a nondecreasing function. On the other hand, if $f(z, t)$ is a continuously differentiable function the above relation is also equivalent to the following ${ }^{[10,11]}$ :

$$
\frac{\mathrm{d} f(z, t)}{\mathrm{d} z} \geqslant 0, \quad \forall z \in \mathbf{R} .
$$

If the function $f(z, t)$ does not satisfy the positivity condition (2), we can introduce a new function $g(z, t)$ such that

$$
g(z, t)=f(z, t)+\rho z, \rho \succ\left\|\frac{\mathrm{d} f(z, t)}{\mathrm{d} z}\right\|, \quad \forall z \in \mathbf{R} .
$$

One can see that

$$
\frac{\mathrm{d} g(z, t)}{\mathrm{d} z}=\frac{\mathrm{d} f(z, t)}{\mathrm{d} z}+\rho \geqslant 0, \quad \forall z \in \mathbf{R} .
$$

In the multivariable case, the sector property can be written as: $z^{\mathrm{T}} f(z, t) \geqslant 0$, here $z$ and $f(z, t)$ are the vectors of appropriate dimension, respectively.

\subsection{Nonlinear observer design}

The circle criterion based nonlinear observer design can be performed for a class of nonlinear systems that can be decomposed into linear part and nonlinear part as the following ${ }^{[10-13]}$ :

$$
\begin{gathered}
\dot{x}(t)=A x(t)+\phi[u(t), y(t)]+G f[H \cdot x(t)] \\
y(t)=C x(t)
\end{gathered}
$$

where $A, C$ and $G$ are known constant matrices with appropriate dimensions. The pair $(A, C)$ is assumed to be observable. The term $\phi[u(t), y(t)]$ is an arbitrary real-valued vector that depends only on the system inputs $u(t)$ and outputs $y(t)$. The nonlinear part of the system is modeled by the term $f[H x(t)]$ which is a time-varying vector function verifying the sector property.

In the following, we recall the main theorem and conditions that are used in this work to study the feasibility of nonlinear observer design for induction motor sensorless control with respect to sector property.

Theorem $\mathbf{1}^{[10,13]}$. Consider a nonlinear system of the form (5) and (6) with the nonlinear part satisfying the circle criterion relations (1)-(4). If there exists a symmetric and positive definite matrix $P \in \mathbf{R}^{n \times n}$ and a set of row vectors $K \in \mathbf{R}^{p}$ such that the following linear matrix inequalities (LMI) hold:

$$
\begin{gathered}
(A-L C)^{\mathrm{T}} P+P(A-L C)+Q \leqslant 0 \\
P G+(H-K C)^{\mathrm{T}}=0 .
\end{gathered}
$$

Then a nonlinear observer can be designed as

$$
\begin{aligned}
\dot{\hat{x}}(t)= & A \hat{x}(t)+\phi[u(t), y(t)]+L[y(t)-\hat{y}(t)]+ \\
& G f[H \hat{x}(t)+K(y(t)-\hat{y}(t))] \\
\hat{y}(t)= & C \hat{x}(t) .
\end{aligned}
$$

And the limit of the state estimation error, $e(t)=x(t)-$ $\hat{x}(t)$, tends to zero when the time instant $t$ tends to infinity. $\hat{x}(t)$ is the estimate of state vector $x(t)$ of the nonlinear system. $Q=\varepsilon I_{n}$ is a known positive defined matrix, $I_{n}$ is an $n$-th order unity matrix, and $\varepsilon$ is a small positive real number.

The nonlinear observer design refers to the selection of the gain matrices $L$ and $K$ satisfying the LMI conditions (7) and (8). One can see that the structure of the nonlinear observer is composed of a linear part, that is similar to linear Luenberger observer, and a nonlinear part that is an additional term that represents the time-varying nonlinearities satisfying the sector property.

The circle criterion based nonlinear observer design takes advantage of the sector property by introducing a nonlinear term in the structure of the observer. In the light of the summary proof presented in [10], we present in the following a detailed proof of the theorem.

Proof. The state estimation error is given as: $e(t)=$ $x(t)-\hat{x}(t)$, where $\hat{x}(t)$ is the estimate of the state vector $x(t)$ of the nonlinear system (5) and (6). The dynamics of the state estimation error are then

$$
\begin{aligned}
\dot{e}(t)= & (A-L C) e(t)+ \\
& G[f(H \cdot x(t))-f(H \cdot \hat{x}(t)+K(y(t)-\hat{y}(t))] .
\end{aligned}
$$

Let $v_{1}=H \cdot x(t)$, and $v_{2}=H \cdot \hat{x}(t)+K(y(t)-\hat{y}(t))$.

By setting $z=v_{1}-v_{2}=(H-K C) e(t)$, the term between brackets in (11) can be seen as a function of the variable $z$, and then $\left[f\left(v_{1}\right)-f\left(v_{2}\right)\right]=f(z, t)$. Taking into account the above result, the error dynamics in (11) can be rewritten as

$$
\begin{gathered}
\dot{e}(t)=(A-L C) e(t)+G f(z, t) \\
z=(H-K C) e(t) .
\end{gathered}
$$

Note that the error dynamics, relations (12) and (13), once again, can be considered as a linear system controlled by a time-varying nonlinear function $f(z, t)$ satisfying the sector property.

Circle criterion establishes that a feedback interconnection of a linear system with a time-varying nonlinearity satisfying the sector property is globally uniformly asymptotically stable ${ }^{[10,13]}$. Based upon the error dynamics, i.e., relations (12) and (13), the nonlinear observer design problem is then equivalent to stabilization of the error dynamics problem.

To this end, a candidate Lyapunov function $V=e^{\mathrm{T}} P e$ is considered. With the help of relation (12) and (13), the derivative of the Lyapunov function becomes

$$
\begin{gathered}
\dot{V}=e^{\mathrm{T}}\left[(A-L C)^{\mathrm{T}} P+P(A-L C)\right] e+ \\
f^{\mathrm{T}}(z, t) G^{\mathrm{T}} P e+e^{\mathrm{T}} P G f(z, t) .
\end{gathered}
$$


By setting

$$
(A-L C)^{\mathrm{T}} P+P(A-L C) \leqslant-Q
$$

and

$$
P G=-(H-K C)^{\mathrm{T}} .
$$

With $Q=\varepsilon I_{n}$ and $\varepsilon \succ 0$, the derivative of the Lyapunov function can be rewritten as

$$
\dot{V} \leqslant-e^{\mathrm{T}} Q e-2 z^{\mathrm{T}} f(z, t) .
$$

The design of nonlinear observer based on circle-criterion approach presents the advantage of removing the global Lipschitz restrictions. However, it introduces linear matrix inequality (LMI) conditions. An extension to multivariable discrete-time case is given in [13] for systems with multiple nonlinearities. In [13], the author has investigated globally Lipschitz systems and bounded-state nonlinear systems. A robust version of the circle-criterion is developed taking into account inputs uncertainties and also measurement noises ${ }^{[14]}$

Bounded-state nonlinear systems constitute a large class of systems that includes electric machine systems. Electric machine models involve the magnetic flux as a key and bounded state variable that is when combined with other state variables of the machine, such as rotor angular velocity, leads to the existence of nonlinear part of the machine model. This is due to the effect of the magnetic material saturation property that is similar to the sector nonlinearity.

\section{Induction motor nonlinear model}

Induction motor, as various other electric machines, constitutes a theoretically interesting and practically important class of nonlinear systems. Induction motor is known as a complex nonlinear system in which time-varying parameters entail additional difficulty for system control and conditions monitoring. Based on the fact that the nonlinear model of the induction motor system can be significantly simplified, if one applies the $d-q$ Park transformation, different structures of the nonlinear model can be investigated and discussed as in [1].

In this paper, the considered nonlinear model of the induction motor is described in stator fixed $d-q$ Park reference frame by the following nonlinear differential equations with the stator current, rotor flux and rotor angular velocity as selected state variables of the machine.

$$
\begin{aligned}
\frac{\mathrm{d}}{\mathrm{d} t} i_{s d} & =-\gamma i_{s d}+\frac{\beta}{T_{r}} \varphi_{r d}+\beta \omega_{r} \varphi_{r q}+\frac{1}{\sigma l_{s}} u_{s d} \\
\frac{\mathrm{d}}{\mathrm{d} t} i_{s q} & =-\gamma i_{s q}-\beta \omega_{r} \varphi_{r d}+\frac{\beta}{T_{r}} \varphi_{r q}+\frac{1}{\sigma l_{s}} u_{s q} \\
\frac{\mathrm{d}}{\mathrm{d} t} \varphi_{r d} & =\frac{m}{T_{r}} i_{s d}-\frac{1}{T_{r}} \varphi_{r d}-\omega_{r} \varphi_{r q} \\
\frac{\mathrm{d}}{\mathrm{d} t} \varphi_{r q} & =\frac{m}{T_{r}} i_{s q}+\omega_{r} \varphi_{r d}-\frac{1}{T_{r}} \varphi_{r q} \\
\frac{\mathrm{d}}{\mathrm{d} t} \omega_{r} & =\alpha\left(\varphi_{r d} i_{s q}-\varphi_{r q} i_{s d}\right)-k_{f} \omega_{r}-k_{l} T_{l}
\end{aligned}
$$

where $\alpha=\frac{n_{p}^{2} m}{J l_{r}}, \beta=\frac{1}{m}\left(\frac{1-\sigma}{\sigma}\right), \sigma=1-\frac{m^{2}}{l_{s} l_{r}}, \gamma=$ $\frac{1}{\sigma}\left(\frac{1}{T_{s}}+\frac{1-\sigma}{T_{r}}\right), k_{f}=\frac{f_{r}}{J}, k_{l}=\frac{n_{p}}{J}$, and $\omega_{r}=n_{p} \Omega_{r}$.

The indices $s$ and $r$ refer to the stator and the rotor components, respectively. The indexes $d$ and $q$ refer to the direct and quadrature of the fixed stator reference frame components, respectively (Park's vector components). $i$ and $u$ are the current and the voltage vector, respectively, $\varphi$ is the flux vector, $r$ is the resistance, $l$ is the inductance, and $m$ is the mutual inductance.

$T_{s}$ and $T_{r}$ are the stator and the rotor time constant, respectively. $\omega_{r}$ is the rotor angular velocity, $f_{r}$ is the friction coefficient, $J$ is the moment of inertia coefficient, $n_{p}$ is the number of pair poles, $\Omega_{r}$ is the mechanical speed of the rotor, and finally $T_{l}$ is the mechanical load torque.

The considered induction motor system model has three inputs and two outputs. Only two state variables are available for measurements which are the stator current components. The nonlinearity of the model is mainly introduced by the product of the rotor angular velocity and the rotor flux components, i.e., relations (18)-(21), and the torque, i.e., relation (22), as the product of two state variables namely the stator current components and the rotor flux components. In order to take into account the effect of the time-varying parameters, such as stator (rotor) resistance, one has to introduce an additional equation relating to the considered parameter variation.

In this paper, we consider only the nonlinearity introduced by the variation of the rotor angular velocity. This type of nonlinear model is generally used for performing nonlinear control, conditions monitoring and faults diagnosis of electric machine systems. Performing these techniques requires the estimation of unmeasured rotor flux linkage and rotor angular velocity based on the stator current and voltage measurements. In this context, the circle criterion approach application is investigated to design a nonlinear observer for induction motor sensorless control.

To satisfy sector conditions (1)-(4), nonlinearities of the machine model (18)-(22) are function of the flux state variable that is a bounded state variable. The nonlinearities of the model are of the form $\omega_{r} \varphi_{r d}$ that can be expressed as

$$
\omega_{r} \varphi_{r d}=\left(\omega_{r} \varphi_{r d}+\rho \omega_{r}\right)-\rho \omega_{r} .
$$

One can verify that

$$
\frac{\partial}{\partial \omega_{r}}\left(\omega_{r} \varphi_{r d}+\rho \omega_{r}\right)=\varphi_{r d}+\rho \geqslant 0 .
$$

With $\left\|\varphi_{r d}\right\| \leqslant 2$, one can choose $\rho=2$.

Once again the system nonlinearity is decomposed into a nonlinearity satisfying the sector property and a linear part to be added to the linear part of the induction motor model.

\section{Simulation results and comments}

Characteristics of the considered induction machine are listed in Table 1.

In order to implement the circle criterion approach, the nonlinear induction motor model, relations (18)-(22), is 
written in the form of model (5) and (6) taking into account properties (3) and (4), with the following notation for the nonlinear term:

$$
G f(H \cdot x)=\sum_{i=1}^{4} G_{i} f_{i}\left(H_{i} x(t)\right)
$$

here the nonlinear functions $f_{i}$ are defined as

$$
\begin{aligned}
& f_{1}\left(H_{1} x(t)\right)=\omega_{r}\left(\varphi_{r q}+\rho\right), f_{2}\left(H_{2} x(t)\right)=\omega_{r}\left(\varphi_{r d}+\rho\right) \\
& f_{3}\left(H_{3} x(t)\right)=i_{s q}\left(\varphi_{r d}+\rho\right), f_{4}\left(H_{4} x(t)\right)=i_{s d}\left(\varphi_{r q}+\rho\right) .
\end{aligned}
$$

With $x(t)=\left[x_{1}, x_{2}, x_{3}, x_{4}, x_{5}\right]^{\mathrm{T}}=\left[i_{s d}, i_{s q}, \varphi_{r d}, \varphi_{r q}, \omega_{r}\right]^{\mathrm{T}}$ as the state vector of the induction machine.

The input-output valued function is defined as $\phi[u(t), y(t))=B u(t)$, where $u(t)=\left[u_{s d}, u_{s q}, T_{l}\right]^{\mathrm{T}}$ is control input of the machine, $B$ is a constant matrix and $y(t)=\left[\begin{array}{ll}i_{s d} & i_{s q}\end{array}\right]^{\mathrm{T}}$ is the measured output vector.

Table 1 Characteristics of the induction motor

\begin{tabular}{ccc}
\hline Symbol & Quantity & Numerical value \\
\hline $\mathrm{P}$ & Power & $1.5 \mathrm{~kW}$ \\
$f$ & Supply frequency & $50 \mathrm{~Hz}$ \\
$U$ & Supply voltage & $220 \mathrm{~V}$ \\
$n_{p}$ & Number of pair poles & 2 \\
$R_{s}$ & Stator resistance & $4.850 \Omega$ \\
$R_{r}$ & Rotor resistance & $3.805 \Omega$ \\
$l_{s}$ & Stator inductance & $0.274 \mathrm{H}$ \\
$l_{r}$ & Rotor inductance & $0.274 \mathrm{H}$ \\
$m$ & Mutual inductance & $0.258 \mathrm{H}$ \\
$\omega_{r}$ & Rotor angular speed & $297.25 \mathrm{rad} / \mathrm{s}$ \\
$J$ & Inertia coefficient & $0.031 \mathrm{~kg}{ }^{2} / \mathrm{s}$ \\
$f_{r}$ & Fiction coefficient & $0.00114 \mathrm{~N} \cdot \mathrm{s} / \mathrm{rad}$ \\
$T_{l}$ & Load torque & $5 \mathrm{~N} \cdot \mathrm{m}$ \\
\hline
\end{tabular}

Taking into account the numerical values of the different parameters of the machine listed in Table 1, one can easily obtain the following numerical model matrices:

$$
\begin{aligned}
& A=\left[\begin{array}{ccccc}
-264.7163 & 0 & 420.9129 & 0 & -60.6204 \\
0 & -264.7163 & 0 & 420.9129 & 60.6204 \\
3.5828 & 0 & -13.8869 & 0 & 2 \\
0 & 3.5828 & 0 & -13.8869 & -2 \\
242.994 & -242.994 & 0 & 0 & -0.0366
\end{array}\right] \\
& B=\left[\begin{array}{ccc}
32.1898 & 0 & 0 \\
0 & 32.1898 & 0 \\
0 & 0 & 0 \\
0 & 0 & 0 \\
0 & 0 & -64.5161
\end{array}\right] \\
& C=\left[\begin{array}{lllll}
1 & 0 & 0 & 0 & 0 \\
0 & 1 & 0 & 0 & 0
\end{array}\right] \\
& G_{1}=\left[\begin{array}{c}
30.3102 \\
0 \\
-1 \\
0 \\
0
\end{array}\right], \quad G_{2}=\left[\begin{array}{c}
0 \\
-30.3102 \\
0 \\
1 \\
0
\end{array}\right]
\end{aligned}
$$

$$
\begin{aligned}
& G_{3}=\left[\begin{array}{c}
0 \\
0 \\
0 \\
0 \\
121.4970
\end{array}\right], \quad G_{4}=\left[\begin{array}{c}
0 \\
0 \\
0 \\
0 \\
-121.4970
\end{array}\right] \\
& H_{1}=H_{2}=\left[\begin{array}{lllll}
0 & 0 & 0 & 0 & 1
\end{array}\right] \\
& H_{3}=\left[\begin{array}{lllll}
0 & 1 & 0 & 0 & 0
\end{array}\right] \\
& H_{4}=\left[\begin{array}{lllll}
1 & 0 & 0 & 0 & 0
\end{array}\right] \text {. }
\end{aligned}
$$

The first step of the simulation consists of resolving the LMI conditions, relation (7) and (8), using an adequate LMI tool such as the LMI tool-box of the Matlab software. The obtained nonlinear observer gain matrices $L$ and $K_{i}$ are the following:

$$
\begin{aligned}
& L=\left[\begin{array}{cc}
-1.6749 & 0.1188 \\
0.1188 & -1.6749 \\
-0.7172 & -0.1075 \\
-0.1075 & -0.7172 \\
1.6201 & -1.6201
\end{array}\right] \\
& K_{1}=\left[\begin{array}{ll}
-1.6037 & -0.7381
\end{array}\right] \\
& K_{2}=\left[\begin{array}{ll}
0.7381 & 1.6037
\end{array}\right] \\
& K_{3}=\left[\begin{array}{ll}
0.3948 & -0.9193
\end{array}\right] \\
& K_{4}=\left[\begin{array}{ll}
-0.9193 & 0.3948
\end{array}\right] .
\end{aligned}
$$

The corresponding Lyapunov matrix for this LMI feasibility test with $\varepsilon=0.04$ is

$$
P=\left[\begin{array}{ccccc}
0.1550 & -0.0710 & 0.0514 & 0.1486 & 0.0274 \\
-0.0710 & 0.1550 & 0.1486 & 0.0514 & -0.0274 \\
0.0514 & 0.1486 & 5.6010 & 0.4659 & -0.0505 \\
0.1486 & 0.0514 & 0.4659 & 5.6010 & 0.0505 \\
0.0274 & -0.0274 & -0.0505 & 0.0505 & 0.0173
\end{array}\right] .
$$

The second step of simulation consists of injecting the obtained numerical values of the gain matrix $L$ and the vectors $K_{i}$ in the observer expression, relation (9) and (10), in which the nonlinear term takes the following form:

$$
\begin{array}{r}
G f(H \cdot \hat{x}(t)+K(y(t)-\hat{y}(t)))= \\
\sum_{i=1}^{4} G_{i} f_{i}\left[H_{i} \cdot \hat{x}(t)+K_{i}(y(t)-\hat{y}(t))\right]
\end{array}
$$

where the vector parameters $G_{i}$ and $H_{i}$ are defined as above as well as the matrices $A, B$ and $C$.

With the help of Matlab $S$-function and Matlab Simulink, the nonlinear machine system and the nonlinear observer are simulated as shown in Fig. 1. Taking into account the following starting conditions $X_{0}=\left[\begin{array}{lllll}4 & 0 & 0 & 0 & 0\end{array}\right]$ for the initial state vector of the machine, the measured and unmeasured state variables of the induction machine are generated. 


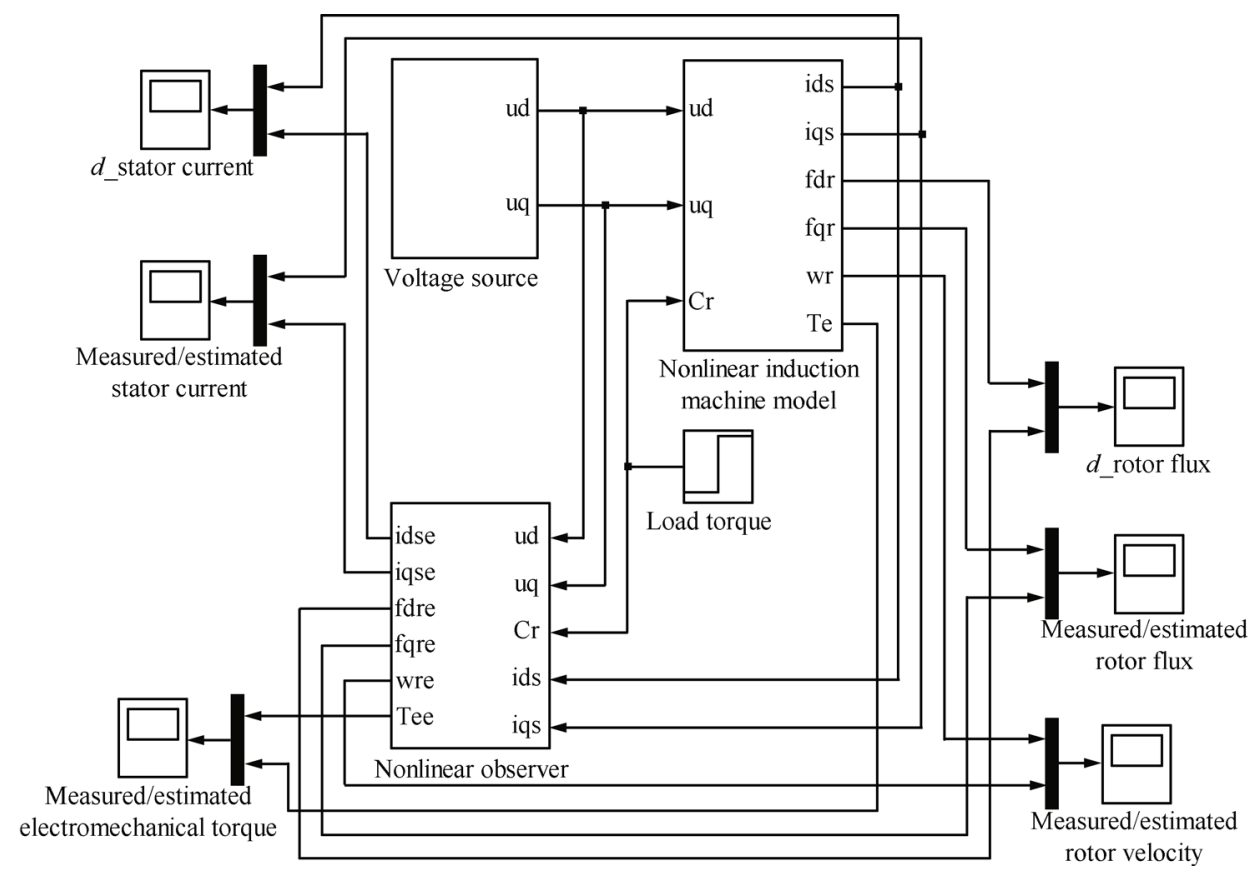

Fig. 1 Matlab-Simulink simulation scheme

The simulation results of the designed nonlinear observer are presented in the following.

Figs. 2 and 3 show the measured (red line) and estimated (blue line) stator current and rotor flux for the $d$-axis and the corresponding error. The $q$-axis components contain the same information as the $d$-axis but they are shifted by $\frac{\pi}{2}$ from the $d$-axis components. (The color figures in this paper can be found in the electronic version.)

Figs. 4 and 5 show the measured (red line) and estimated (blue line) rotor angular velocity and the electromechanical torque, respectively, with the corresponding estimation error. One can see that the estimated state variables of the machine follow the desired trajectories.
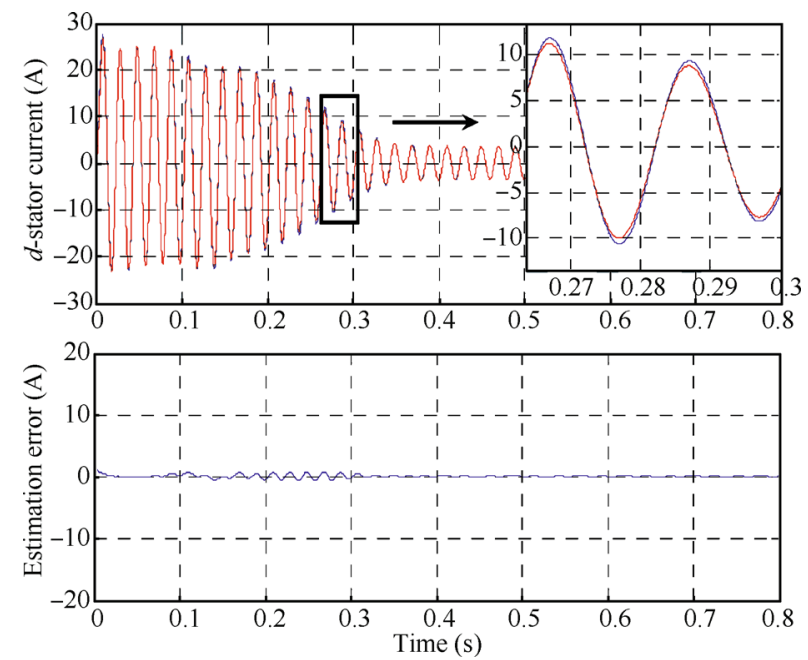

Fig. 2 Measured (red line) and observed (blue line) $d$-stator current components and the corresponding estimation error
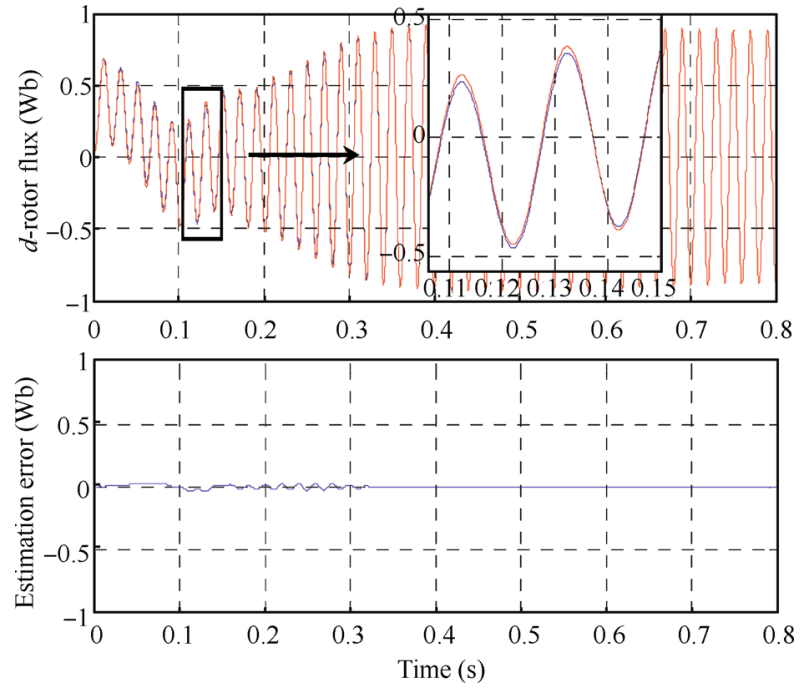

Fig. 3 Measured (red line) and observed (blue line) $d$-rotor flux component and the corresponding estimation error

For further clarity, a zoomed part is added in each figure to show the difference between the measured and the estimated state variables of the induction machine system.

One can see that the estimation error is null after a transient state. Thus it confirms that the designed nonlinear observer, based on the circle criterion, estimates effectively the unmeasured state variables of the considered induction machine. Measured and estimated state variables of the considered induction machine can be used to control the machine system via an adequate state feedback control technique.

Evaluation of the performance of the designed observer in the low speed region and even at zero speed, with and without load torque, is a research area that effectively opens 
a large variety of applications of sensorless induction motor drive, which will be investigated in the forthcoming paper.
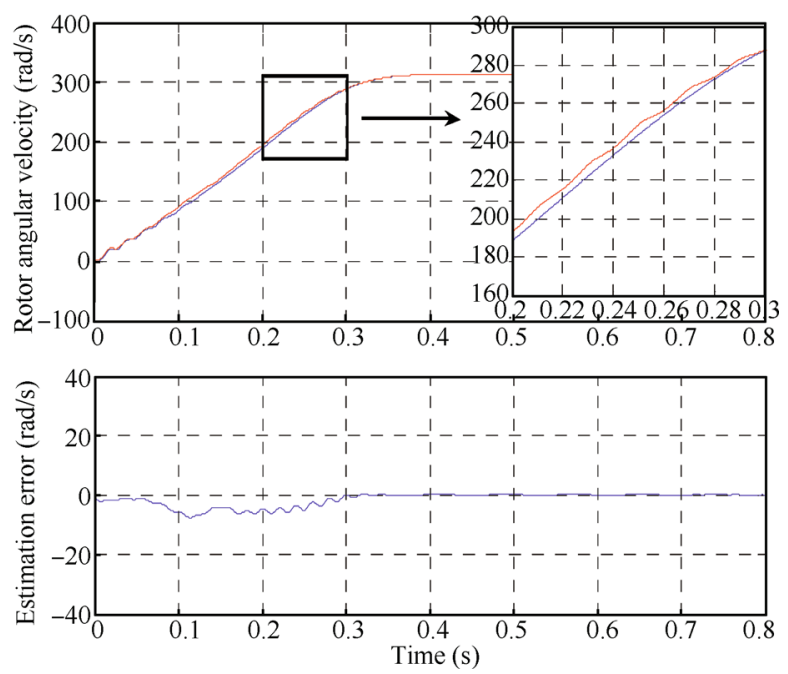

Fig. 4 Measured (red line) and observed (blue line) rotor angular velocity and the corresponding estimation error
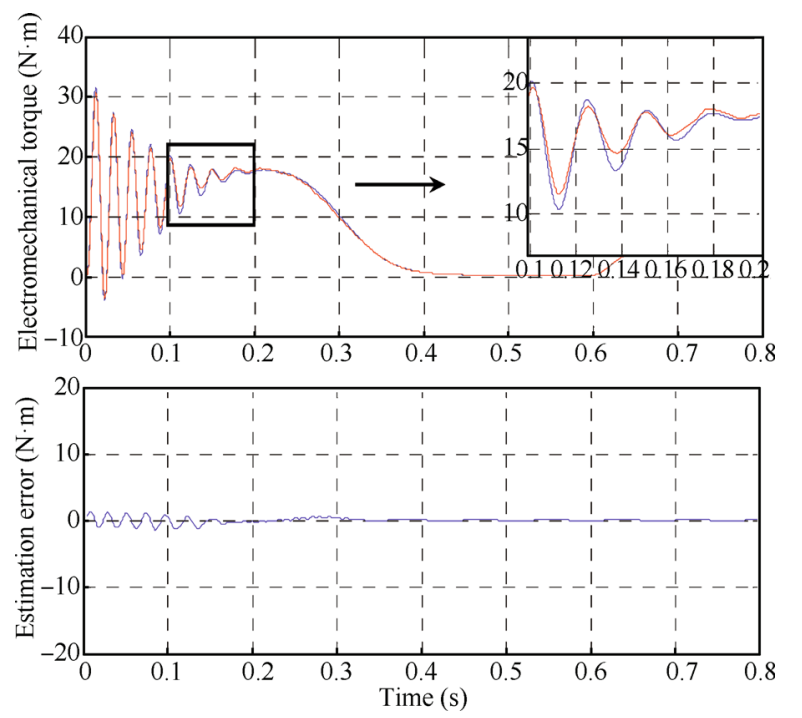

Fig. 5 Measured (red line) and estimated (blue) electromechanical torque of the machine and the corresponding estimation error

\section{Conclusions}

A circle criterion based nonlinear observer design for induction motor sensorless control has been presented.

The main advantage of the circle criterion approach is that it permits to exploit directly the nonlinearities of the system without attempting to eliminate them. However, it introduces linear matrix inequalities as conditions for the convergence of the observer and the error of state estimation. Resolving the LMI determines the gain matrices of the nonlinear observer.

Simulation results show that the circle criterion based nonlinear observer design can effectively be performed to estimate unmeasurable state variables of the induction ma- chine for possible sensorless control.

\section{References}

[1] B. Bensaker, H. Kherfane, A. Maouche, R. Wamkeue. Nonlinear modeling of induction motor drives for nonlinear sensorless control purposes. In Proceedings of the 6th IFAC Symposium on Nonlinear Control Systems, IFAC, Stuttgart, Germany, vol. 3, pp. 1475-1480, 2004.

[2] M. G. Campbell, J. Chiasson, M. Bodson, L. M. Tolbert. Speed sensorless identification of the rotor time constant in induction machines. IEEE Transactions on Automatic Control, vol. 52, no. 4, pp. 758-763, 2007.

[3] R. Yazdanpanah, J. Soltani, G. R. A. Markadeh. Nonlinear torque and stator flux controller for induction motor drive based on adaptive input-output feedback linearization and sliding mode control. Energy Conversion and Management, vol. 49, pp. 541-550, 2008.

[4] A. N. Atassi, H. K. Khalil. Separation results for the stabilization of nonlinear systems using different high-gain observer designs. Systems \& Control Letters, vol. 39, no. 3, pp. 183-191, 2000.

[5] N. Boizot, E. Busvelle, J. P. Gauthier. An adaptive highgain observer for nonlinear systems. Automatica, vol. 46, no. 9, pp. $1483-1488,2010$.

[6] A. Zemmouche, M. Boutaeib. On LMI conditions to design observers for Lipschitz nonlinear systems. Automatica, vol. 49, no. 2, pp. 585-591, 2013.

[7] H. Beikzadeh, H. D. Taghirad. Exponential nonlinear observer based on the differential state-dependent Riccati equation. International Journal of Automation and Computing, vol. 9, no. 4, pp. 358-368, 2012.

[8] L. P. Liu, Z. M. Fu, X. N. Song. Sliding mode control with disturbance observer for a class of nonlinear systems. International Journal of Automation and Computing, vol. 9, no. 5, pp. 487-491, 2012.

[9] J. Chen, E. Prempain, Q. H. Wu. Observer-based nonlinear control of a torque motor with perturbation estimation. International Journal of Automation and Computing, vol. 3, no. 1, pp. 84-90, 2006.

[10] M. Arcak, P. Kokotovic. Nonlinear observers: A circle criterion design and robustness analysis. Automatica, vol. 37, no. 12, pp. 1923-1930, 2001.

[11] M. Arcak. Certainty-equivalence output-feedback design with circle-criterion observers. IEEE Transactions on $\mathrm{Au}$ tomatic Control, vol. 50, no. 6, pp. 905-909, 2005.

[12] W. Bourbia, F. Berrezzek, B. Bensaker. A Circle-criterion based nonlinear observer design for induction motor conditions monitoring. In Proceedings of the 7th European Nonlinear Dynamics Conference, Roma, Italy, 2011.

[13] S. Ibrir. Circle-criterion approach to discrete-time nonlinear observer design. Automatica, vol. 43, no. 8, pp. 1432-1441, 2007.

[14] M. Chong, R. Postoyan, D. Nesic, L. Kuhlmann, A Varsavsky. A robust circle criterion observer with application to neural mass models. Automatica, vol. 48, no. 11, pp. 2986-2989, 2012. 


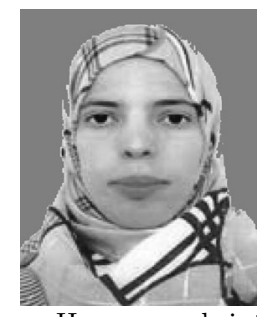

Wafa Bourbia graduated from University of Badji Mokhtar Annaba (UBMA), Algeria. She received her B. Sc. and M.Sc. degrees in electrotechnical engineering from the UBMA in 2003 and 2006, respectively. Currently, she is involved in doctorate studies on nonlinear sensorless control of electric machine systems. She has published a few refereed conference papers.

Her research interests include system control techniques and their application to electric machines.

E-mail: bourbia_wafa@yahoo.fr

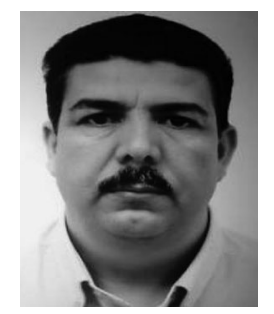

Farid Berrezzek graduated from University Badji Mokhtar Annaba (UBMA), Algeria. He received his B.Sc. and M.Sc. degrees in electrotechnical engineering from the UBMA in 1994 and 2006, respectively. Currently, he is involved in doctorate studies on nonlinear control of electric machine systems. He has published a few refereed conference papers.
His research interests include system control techniques and their application to electric machines.

E-mail: berrezzek_farid@yahoo.fr

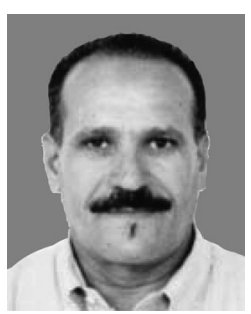

Bachir Bensaker graduated from University of Science and Technology of Oran (USTO), Algeria. He received his B.Sc. degree in electronics engineering in 1979. He received his M.S. and Ph. D. degrees in instrumentation and control from the Universities of Rouen and Le Havre, France, in 1985 and in 1988, respectively. Since 1988 , he has been with the Department of Electronics, University of Annaba (UBMA), Algeria, where, since 2004, he has been a full professor. He has been an IFAC affiliate since 1991 . He has published about 50 refereed journal and conference papers.

His research interests include system modelling, control, identification, estimation, and system reliability and their applications in nonlinear control, condition monitoring, fault detection and diagnostics of electrical machines.

E-mail: bensaker_bachir@yahoo.fr (Corresponding author) 